\title{
Identification of key genes for diabetic kidney disease using biological informatics methods
}

\author{
FUZHE MA, TAO SUN, MEIYAN WU, WANNING WANG and ZHONGGAO XU \\ Department of Nephrology, The First Hospital of Jilin University, Changchun, Jilin 130021, P.R. China
}

Received December 4, 2016; Accepted June 11, 2017

DOI: $10.3892 / \mathrm{mmr} .2017 .7666$

\begin{abstract}
Diabetic kidney disease (DKD) is a common complication of diabetes, which is characterized by albuminuria, impaired glomerular filtration rate or a combination of the two. The aim of the present study was to identify the potential key genes involved in DKD progression and to subsequently investigate the underlying mechanism involved in DKD development. The array data of GSE30528 including 9 DKD and 13 control samples was downloaded from the Gene Expression Omnibus database. The differentially expressed genes (DEGs) in DKD glomerular and tubular kidney biopsy tissues were compared with normal tissues, and were analyzed using the limma package. Gene Ontology (GO) annotation and Kyoto Encyclopedia of Genes and Genomes (KEGG) pathway enrichment analyses were performed for DEGs using the GO Function software in Bioconductor. The protein-protein interaction (PPI) network was then constructed using Cytoscape software. A total of 426 genes (115 up- and 311 downregulated) were differentially expressed between the DKD and normal tissue samples. The PPI network was constructed with 184 nodes and 335 edges. Vascular endothelial growth factor A (VEGFA), $\alpha$-actinin-4 (ACTN4), proto-oncogene, Src family tyrosine kinase (FYN), collagen, type 1, $\alpha 2$ (COL1A2) and insulin-like growth factor 1 (IGF1) were hub proteins. Major histocompatibility complex, class II, DP $\alpha 1$ (HLA-DPA1) was the common gene enriched in the rheumatoid arthritis and systemic lupus erythematosus pathways, and the immune response was a GO term enriched in module A. VEGFA, ACTN4, FYN, COL1A2, IGF1 and $H L A-D P A 1$ may be potential key genes associated with the progression of DKD, and immune mechanisms may serve a part in DKD development.
\end{abstract}

Correspondence to: Dr Zhonggao Xu, Department of Nephrology, The First Hospital of Jilin University, 71 Xinmin Street, Changchun, Jilin 130021, P.R. China

E-mail: zhonggaoxu@hotmail.com

Key words: diabetic kidney disease, differentially expressed genes, Kyoto Encyclopedia of Genes and Genomes pathway, protein-protein interaction network, key genes

\section{Introduction}

Diabetic kidney disease (DKD) is a common complication of diabetes, which is characterized by albuminuria, an impaired glomerular filtration rate (GFR) or a combination of the two $(1,2)$. DKD accounts for $\sim 50 \%$ of all cases of end-stage renal disease (ESRD) in the United States and the US ESRD program is a large medical expense/economic burden and costs a great amount of money to run. However, a number of genes closely associated with DKD development have yet to be identified despite many years of intensive study (3). Therefore, the identification of genes associated with DKD development is urgently required, as well as the subsequent elucidation of its molecular mechanism.

Some advancements have been made in the elucidation of the pathological mechanism involved in the development of DKD. The loss of podocytes is an early feature of DKD (4). The levels of almost all podocyte-specific genes including genes for congenital nephrotic syndrome of the finish type (NPHS1), glomerular podocin (NPHS2), the Wilm's tumor gene (WT1) and vascular endothelial growth factor $(V E G F)$ are all severely reduced in DKD (3). Some other studies have also demonstrated that NPHS1 (5,6), NPHS2 (7), bone morphogenetic protein 7 (8), WT1 (4) and VEGF $(9,10)$ are decreased in DKD. In addition, tubulointerstitial fibrosis is a prominent feature of progressive DKD and is likely to be one of the final common pathways leading to the development of ESRD, with patients subsequently requiring dialysis or transplantation $(11,12)$. A previous study revealed that using angiotensin-converting-enzyme-inhibitors and angiotensin II receptor antagonists in patients with diabetes mellitus can respectively improve mortality and delay the progression of DKD (13). In addition, a human genetic study highlighted that the complement system potentially serves a role in low-grade inflammation and the development of DKD (14). Therefore, the aim of the present study was to identify the key genes associated with the development of DKD and elucidate its underlying mechanism.

In the present study, the microarray data of GSE30528 was downloaded the from Gene Expression Omnibus database (GEO; www.ncbi.nlm.nih.gov/geo/). The gene expression profiles in DKD were analyzed and functional analysis was performed for differentially expressed genes (DEGs) in DKD glomerular and tubular kidney biopsy tissues in comparison with normal tissues. In addition, the protein-protein interaction (PPI) network was also constructed. These results were used to 
discover the key genes associated with DKD development and to clarify the underlying mechanism.

\section{Materials and methods}

Affymetrix microarray data. The array data for GSE30528 was downloaded from the GEO database, which was first recorded by Woroniecka et al (3) and was based on the GPL571 platform (Affymetrix Human Genome U133A 2.0 Array; Affymetrix, Inc.; Thermo Fisher Scientific, Inc., Waltham, MA, USA). A total of 44 samples were used to develop the original array data, and of these 9 DKD [age, $64 \pm 13.56$ years; 5 females, 4 males; body mass index (BMI), $\left.32.74 \pm 7.9 \mathrm{~kg} / \mathrm{m}^{2}\right]$ and 13 healthy, disease-free control samples (age, $51.38 \pm 12.01$ years; 5 females, 8 males; BMI, $29.59 \pm 9.08 \mathrm{~kg} / \mathrm{m}^{2}$ ) were selected for analysis in the present study.

Data processing and DEG analysis. The raw expression data was preprocessed using the robust multiarray average algorithm (15) and the Affy package in Bioconductor (bioconductor .org/packages/release/bioc/html/affy.html); the expression levels of the probes were then obtained. If several probes mapped to one gene symbol, then the mean value was set as the final expression value of this gene. The DEGs in DKD glomerular and tubular kidney biopsy tissues where then compared with normal tissues using the limma package (16). $\log \mathrm{FCl}>1$ and $\mathrm{P}<0.05$ were considered as the cutoff criterion.

Gene Ontology (GO) and pathway enrichment analysis. GO is used for the unification of biology, collecting defined, structured and controlled vocabulary for gene annotation, which mainly includes the following 3 categories: Molecular function, biological process and cellular component (17). The Kyoto Encyclopedia of Genes and Genomes (KEGG) is a database for the classification of relevant gene sets into their respective pathways (18).

In order to analyze the DEGs on a functional level, GO annotation and KEGG pathway enrichment analyses were performed for DEGs using GO Function version 1.24.0 (19) software in Bioconductor version 3.5 (www.bioconductor. org/packages/release/bioc/html/GOFunction.html), and gene annotation information was obtained from the org. Hs. eg. db and GO. db package. $\mathrm{P}<0.05$ and gene counts $>2$ were set as the cut off values.

PPI network analysis. The Search Tool for the Retrieval of Interacting Genes (STRING) database provides the experimental and predicted interaction information of proteins (20). Protein pair interactions in STRING were presented with a combined score. The DEGs were mapped into PPIs and a combined score of $>0.7$ was identified as the cutoff standard for the important protein pairs. The PPI network was constructed using Cytoscape software version 2.8.2 (www.cytoscape.org/) (21).

Module analysis. ClusterONE version 1.0 (www.paccanarolab. org/cluster-one/) in the Cytoscape software package was used to analyze the PPI network modules with a minimum size of 3 and a minimum density of 0.5 . Modules with $\mathrm{P}<0.01$ were set as significant clustering modules.

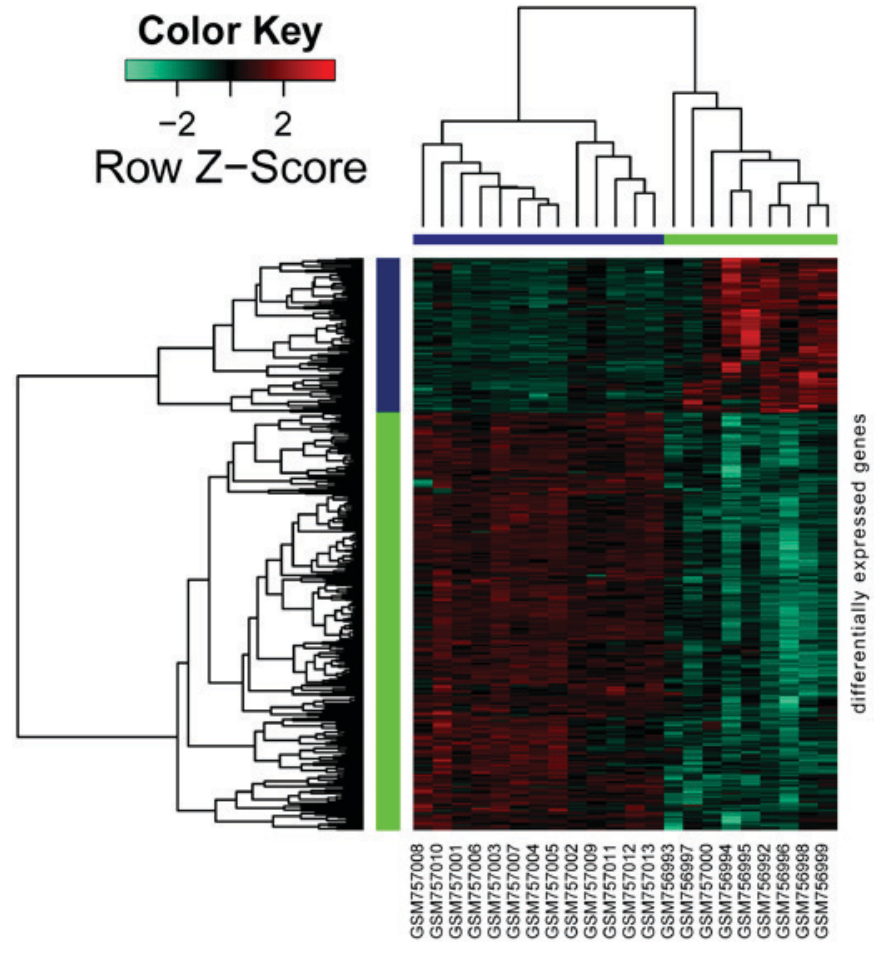

Figure 1. A heat map of the differentially expressed genes identified. Horizontal band with the tree at the top: blue, normal samples; green, diabetes samples; vertical band with the tree on the side: blue, upregulated genes; green, downregulated genes.

\section{Results}

Data processing and DEGs analysis. As shown in Fig. 1, a total of 426 genes were differentially expressed in DKD samples when compared with normal samples, amongst which 115 genes were upregulated and 311 were downregulated.

GO and pathway enrichment analysis. GO and KEGG pathway analyses were performed for upregulated and downregulated DEGs. The top 5 GO terms are shown in Table I. The overrepresented GO terms of upregulated DEGs were primarily associated with extracellular region, antigen binding, extracellular space, the defense response, the immune response and peptidase regulator activity (Table IA). The downregulated DEGs were mainly involved in cardiovascular system development, circulatory system development, actin cytoskeleton, cell junction, cytoskeletal protein binding and integrin binding (Table IB).

The upregulated DEGs were mainly enriched in 17 KEGG pathways, including primary immunodeficiency, extracellular matrix-receptor interactions, rheumatoid arthritis and systemic lupus erythematosus (Table IIA). In addition, major histocompatibility complex, class II, DP $\alpha 1$ (HLA-DPA1) was the common gene in the rheumatoid arthritis and systemic lupus erythematosus pathways. The downregulated DEGs were mainly enriched in $16 \mathrm{KEGG}$ pathways, such as tight junction and adherens junction.

PPI network analysis. Based on the STRING database, a total of 335 protein pairs with a combined score of $>0.7$ were obtained. 
Table I. Gene Ontology analysis for differentially expressed genes.

A, Upregulated

\begin{tabular}{|c|c|c|c|}
\hline Term & Description & Counts (n) & P-value \\
\hline \multicolumn{4}{|l|}{ GO-BP terms } \\
\hline GO:0006952 & Defense response & 44 & $<0.0005$ \\
\hline GO:0006955 & Immune response & 41 & $2.22 \times 10^{-16}$ \\
\hline GO:0002376 & Immune system process & 50 & $3.00 \times 10^{-15}$ \\
\hline GO:0001775 & Cell activation & 31 & $1.37 \times 10^{-14}$ \\
\hline GO:0045321 & Leukocyte activation & 26 & $1.76 \times 10^{-13}$ \\
\hline \multicolumn{4}{|l|}{ GO-CC terms } \\
\hline GO:0005576 & Extracellular region & 63 & $2.06 \times 10^{-12}$ \\
\hline GO:0005615 & Extracellular space & 32 & $4.37 \times 10^{-12}$ \\
\hline GO:0031982 & Vesicle & 53 & $9.90 \times 10^{-11}$ \\
\hline GO:0031988 & Membrane-bounded vesicle & 52 & $1.13 \times 10^{-10}$ \\
\hline GO:0044421 & Extracellular region part & 53 & $3.50 \times 10^{-10}$ \\
\hline \multicolumn{4}{|l|}{ GO-MF terms } \\
\hline GO:0003823 & Antigen binding & 8 & $2.97 \times 10^{-07}$ \\
\hline GO:0061134 & Peptidase regulator activity & 10 & $6.67 \times 10^{-07}$ \\
\hline GO:0005539 & Glycosaminoglycan binding & 9 & $2.84 \times 10^{-07}$ \\
\hline GO:0004866 & Endopeptidase inhibitor activity & 8 & \\
\hline \multicolumn{4}{|l|}{$8092 \times 10^{-06}$} \\
\hline GO:0061135 & Endopeptidase regulator activity & 8 & $1.11 \times 10^{-05}$ \\
\hline
\end{tabular}

B, Downregulated

\begin{tabular}{clcl}
\hline Term & \multicolumn{1}{c}{ Description } & Counts (n) & P-value \\
\hline GO-BP terms & & & \\
GO:0072358 & Cardiovascular system development & 50 & $3.77 \times 10^{-15}$ \\
GO:0072359 & Circulatory system development & 50 & $3.77 \times 10^{-15}$ \\
GO:0009653 & Anatomical structure morphogenesis & 91 & $4.66 \times 10^{-15}$ \\
GO:0048731 & System development & 121 & $3.40 \times 10^{-14}$ \\
GO:0032502 & Developmental process & 147 & $5.66 \times 10^{-14}$ \\
GO-CC terms & & & \\
GO:0015629 & Actin cytoskeleton & 31 & $1.79 \times 10^{-12}$ \\
GO:0030054 & Cell junction & 47 & $6.04 \times 10^{-10}$ \\
GO:0070161 & Anchoring junction & 28 & $1.82 \times 10^{-09}$ \\
GO:0005912 & Adherens junction & 27 & $3.39 \times 10^{-09}$ \\
GO:0044421 & Extracellular region part & 98 & $3.00 \times 10^{-08}$ \\
GO-MF terms & & & \\
GO:0008092 & Cytoskeletal protein binding & 40 & $9.40 \times 10^{-11}$ \\
GO:0005178 & Integrin binding & 12 & $1.23 \times 10^{-07}$ \\
GO:0032403 & Protein complex binding & 35 & $4.24 \times 10^{-07}$ \\
GO:0050839 & Cell adhesion molecule binding & 14 & $8.04 \times 10^{-07}$ \\
GO:0003779 & Actin binding & 21 & $1.40 \times 10^{-06}$ \\
& & &
\end{tabular}

${ }^{\mathrm{a}} \mathrm{P}<0.00001$ vs. normal matched tissues. GO, Gene Ontology; BP, biological process; CC, cellular component; MF, molecular function; Term, the identification number of GO; Description, the name of the GO term; Counts, the number of genes enriched in the GO term.

As presented in Fig. 2, the PPI network was constructed with 335 edges and 184 nodes. The nodes of VEGFA (degree score, 19), $\alpha$-actinin-4 (ACTN4; degree score, 17), proto-oncogene, Src family tyrosine kinase (FYN; degree score, 17), collagen, type 
Table II. Kyoto Encyclopedia of Genes and Genomes pathway enrichment analysis of the top 10 DEGs.

A, Upregulated

\begin{tabular}{llrr}
\hline Term & \multicolumn{1}{c}{ Description } & Counts $(\mathrm{n})$ & P-value \\
\hline 5150 & Staphylococcus aureus infection & 5 & $1.89 \times 10^{-04 \mathrm{c}}$ \\
5020 & Prion diseases & 4 & $3.58 \times 10^{-04 \mathrm{c}}$ \\
5340 & Primary immunodeficiency & 4 & $3.58 \times 10^{-04 \mathrm{c}}$ \\
4512 & Extracellular matrix-receptor interaction & 5 & $1.42 \times 10^{-03 \mathrm{~b}}$ \\
5323 & Rheumatoid arthritis & 5 & $1.92 \times 10^{-03 \mathrm{~b}}$ \\
5142 & Chagas disease (American trypanosomiasis) & 5 & $3.45 \times 10^{-03 \mathrm{~b}}$ \\
4610 & Complement and coagulation cascades & 4 & $4.61 \times 10^{-03 \mathrm{~b}}$ \\
4974 & Protein digestion and absorption & 4 & $8.12 \times 10^{-03 \mathrm{~b}}$ \\
5322 & Systemic lupus erythematosus & 5 & $1.06 \times 10^{-02 \mathrm{a}}$ \\
4640 & Hematopoietic cell lineage & 4 & $1.08 \times 10^{-02 \mathrm{a}}$ \\
\hline
\end{tabular}

B, Downregulated

\begin{tabular}{llrr}
\hline Term & \multicolumn{1}{c}{ Description } & Counts (n) & P-value \\
\hline 4520 & Adherens junction & 8 & $3.85 \times 10^{-05 d}$ \\
4510 & Focal adhesion & 13 & $4.51 \times 10^{-05 \mathrm{~d}}$ \\
4810 & Regulation of actin cytoskeleton & 9 & $8.65 \times 10^{-05 \mathrm{~d}}$ \\
4530 & Tight junction & 7 & $5.06 \times 10^{-04 \mathrm{c}}$ \\
5410 & Hypertrophic cardiomyopathy & 7 & $6.17 \times 10^{-04 \mathrm{c}}$ \\
5414 & Dilated cardiomyopathy & 6 & $1.00 \times 10^{-03 \mathrm{~b}}$ \\
5412 & Arrhythmogenic right ventricular cardiomyopathy & 5 & $1.87 \times 10^{-03 \mathrm{~b}}$ \\
4610 & Complement and coagulation cascades & 7 & $7.26 \times 10^{-03 \mathrm{~b}}$ \\
4360 & Axon guidance & 12 & $7.65 \times 10^{-03 \mathrm{~b}}$ \\
5200 & Pathways in cancer & $1.21 \times 10^{-02 \mathrm{a}}$ &
\end{tabular}

${ }^{\mathrm{a}} \mathrm{P}<0.05,{ }^{\mathrm{b}} \mathrm{P}<0.01,{ }^{\mathrm{C}} \mathrm{P}<0.001$ and ${ }^{\mathrm{d}} \mathrm{P}<0.0001$ vs. normal matched tissues. KEGG, Kyoto Encyclopedia of Genes and Genomes; Term, the identification number of the KEGG pathway; Description, the name of the KEGG pathway; Counts, the number of genes enriched in the KEGG pathway.

$1, \alpha 2$ (COL1A2; degree score, 15) and insulin-like growth factor 1 (IGF1; degree score, 15) were hub proteins in the network.

Modules analysis. Two significant clustering modules were obtained using ClusterONE software (Fig. 3). A total of 8 and 6 nodes were enriched in modules $\mathrm{A}$ and $\mathrm{B}$, respectively. As shown in Table III, nodes in module A (density, 1.0; quality, $0.800 ; \mathrm{P}=1.606 \times 10^{-4}$ ) were mainly enriched in GO: G-protein coupled receptor protein signaling pathway, cell surface receptor linked signal transduction, the immune response, the chemokine signaling pathway and the cytokine-cytokine receptor interaction pathway. Nodes in module B (density, 1.0; quality, 0.789; $\mathrm{P}=0.001$ ) were mainly enriched in $\mathrm{GO}$ : regulation of ATPase activity, regulation of system process and regulation of hydrolase activity.

\section{Discussion}

In the present study, using the gene expression patterns downloaded from the GEO database, 426 DEGs in DKD glomerular and tubular kidney biopsy tissues were obtained and compared with matched normal tissues, identifying 115 upregulated genes and 311 downregulated DEGs. The results demonstrated that HLA-DPAl was the common gene enriched in the rheumatoid arthritis and systemic lupus erythematosus pathways, and the immune response was a significant GO term enriched in module A. In addition, VEGFA, ACTN4, FYN, COLIA2 and IGF1 had higher degrees and were established as hub nodes in the PPI network; they may therefore contribute to the progression of DKD.

A previous study suggested that cells in the immune system may be involved in the progression of DKD (22). Immune cells take part in vascular injury under DKD-associated conditions (23). Other previous studies have also indicated that the immune system is associated with DKD development (24-26). Primary immunodeficiency (27), rheumatoid arthritis (28) and systemic lupus erythematosus (29) are associated with the immune system. In the present study, primary immunodeficiency, rheumatoid arthritis and systemic lupus erythematosus were 3 significantly enriched pathways, and the immune response was a GO term enriched in module A. Thus, the results of the present study are in agreement with previous findings, 


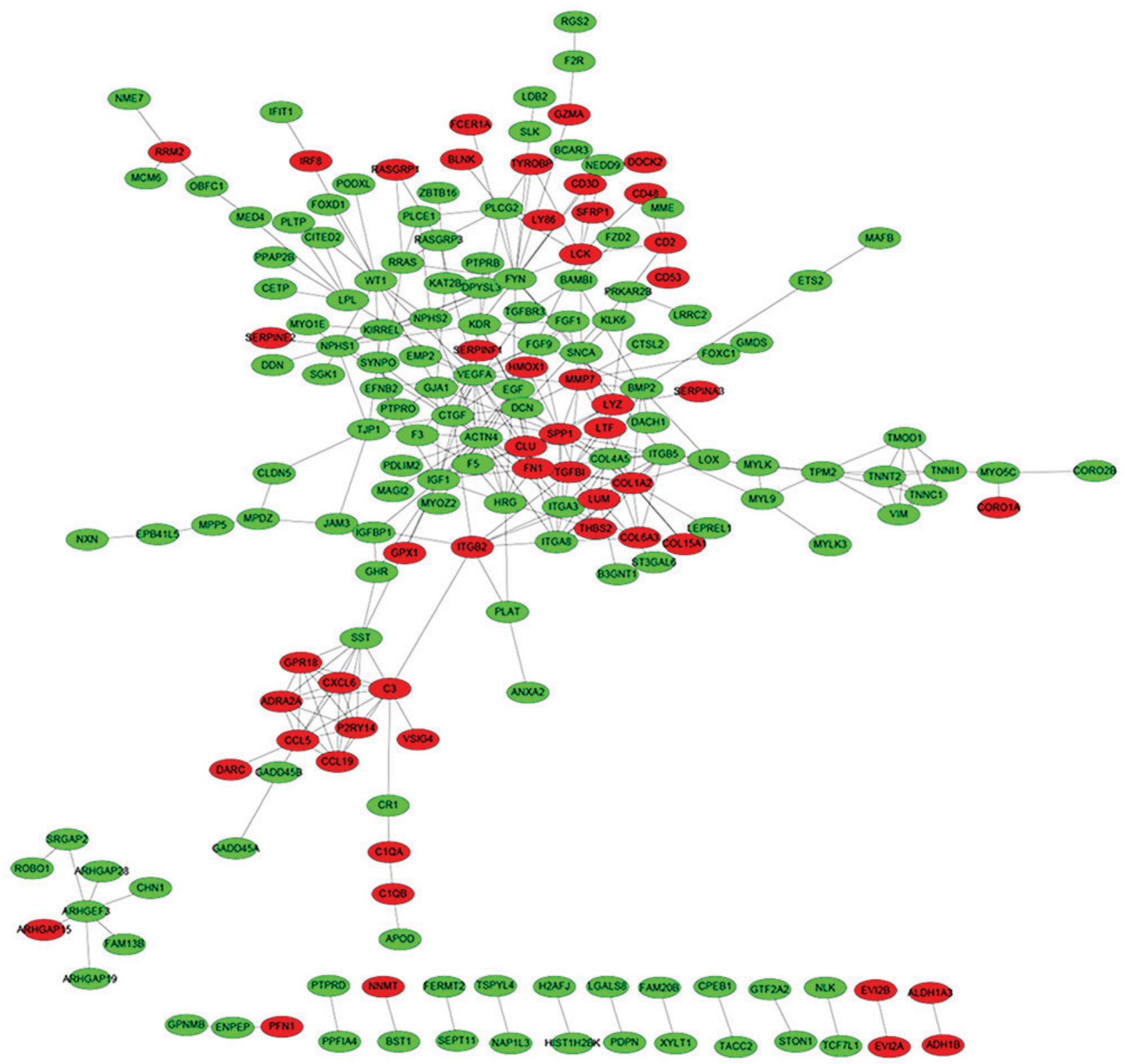

Figure 2. Protein-protein interaction networks of differentially expressed genes. The red nodes represent the upregulated genes and the green nodes represent the downregulated genes.

and therefore indicate that immune mechanisms may serve a role in DKD development.

The work of Woroniecka et al (3) suggested that HLA-DPA1 was a differentially expressed transcript in the tubulointerstitium of patients with DKD when compared with normal samples. Previous studies revealed that $H L A-D P A 1$, which is the closest centromeric gene expressed to HLA-DO $\alpha$, may contribute to the differences in the associated risks of diabetes $(30,31)$, including DKD, which is a complication of diabetes. In the present study, HLA-DPAl was the common gene enriched in the rheumatoid arthritis and systemic lupus erythematosus pathways. Therefore, these results are in line with previous findings and suggest that HLA-DPA1 may contribute to DKD development.

In addition, VEGFA, ACTN4, FYN, COL1A2 and IGF1 were identified as hub proteins in the PPI network. VEGFA is an important angiogenic growth factor that regulates endothelial cells' permeability and vasculogenesis (32). It is also important for the differentiation, proliferation, survival and migration of endothelial cells within the glomerulus (33). Previous studies have suggested that VEGFA may serve a significant role in retaining glomerular endothelial cell function as a reduction in VEGFA levels induced abnormal remodeling of glomerular capillaries $(34,35)$. VEGF may also serve a role in the pathogenesis of DKD (36) and the dysregulation of VEGFA may serve a pathogenic role in inducing glomerular injury (37). In DKD, VEGFA has reduced mRNA expression and may be a potential factor that can lead to the development of DKD by inducing microvascular rarefaction and tubular atrophy (9). In addition, neoangiogenesis, which is caused by overexpression of VEGFA, may stimulate the development of DKD and therefore blocking VEGFA or its signaling may ameliorate DKD (38). These findings indicate that VEGFA may serve a role in DKD progression.

ACTNs are actin-binding proteins that are critical in cell adhesion and in the organization of the cytoskeleton (39). 
Table III. Functional enrichment analysis of protein-protein interaction network clustering modules.

\begin{tabular}{llrl}
\hline Term & \multicolumn{1}{c}{ Description } & Counts (n) & P-value \\
\hline Module A & & 8 & $2.67 \times 10^{-08 a}$ \\
GO_BP:0007186 & G-protein coupled receptor protein signaling pathway & 8 & $9.06 \times 10^{-07 a}$ \\
GO_BP:0007166 & Cell surface receptor linked signal transduction & 5 & $2.08 \times 10^{-04 \mathrm{~b}}$ \\
GO_BP:0006955 & Immune response & 3 & $1.25 \times 10^{-02 \mathrm{c}}$ \\
KEGG_hsa04062 & Chemokine signaling pathway & 3 & $2.38 \times 10^{-02 \mathrm{c}}$ \\
KEGG_hsa04060 & Cytokine-cytokine receptor interaction & 3 & \\
Module B & & 3 & 3 \\
GO_BP:0043462 & Regulation of ATPase activity & 2 & $4.31 \times 10^{-05 \mathrm{~d}}$ \\
GO_BP:0044057 & Regulation of system process & $3.89 \times 10^{-03 \mathrm{e}}$ \\
GO_BP:0051336 & Regulation of hydrolase activity & $2.32 \times 10^{-04 \mathrm{~b}}$ \\
KEGG_hsa04260 & Cardiac muscle contraction & $2.76 \times 10^{-04 \mathrm{~b}}$ \\
KEGG_hsa05410 & Hypertrophic cardiomyopathy & $3.24 \times 10^{-04 \mathrm{~b}}$ \\
KEGG_hsa05414 & Dilated cardiomyopathy & \multicolumn{1}{c}{} \\
\hline
\end{tabular}

${ }^{\mathrm{a}} \mathrm{P}<0.00001,{ }^{\mathrm{b}} \mathrm{P}<0.0005,{ }^{\mathrm{c}} \mathrm{P}<0.05,{ }^{\mathrm{d}} \mathrm{P}<0.0001,{ }^{\mathrm{e}} \mathrm{P}<0.005$ and ${ }^{\mathrm{f}} \mathrm{P}<0.01$ vs. normal matched tissues. GO, Gene Ontology; KEGG, KEGG, Kyoto Encyclopedia of Genes and Genomes; Term, the identification number of GO-Biological Process or KEGG pathway; Description, the name of the GO- Biological Process or KEGG pathway; Counts, the number of genes enriched in GO- Biological Process or KEGG pathway.
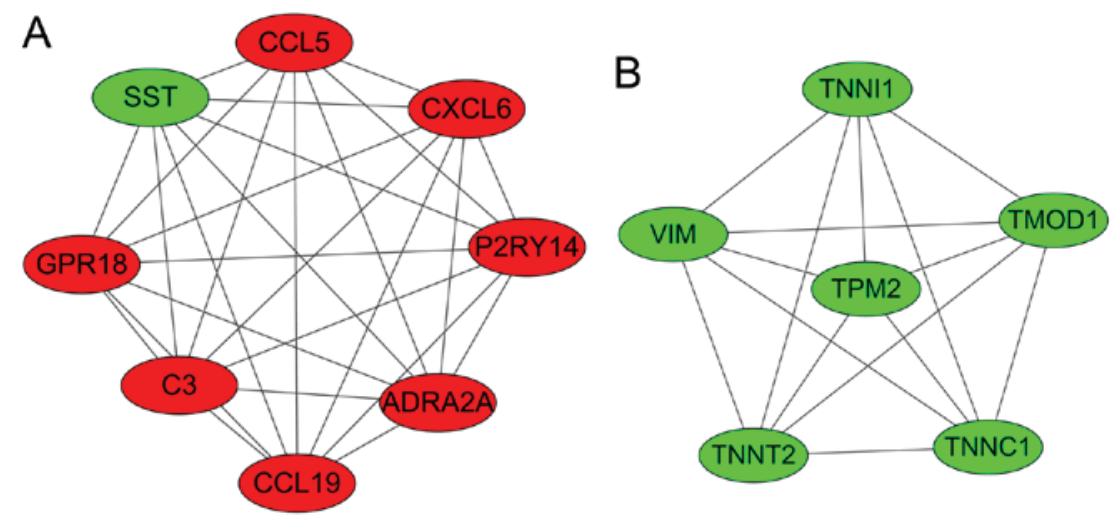

Figure 3. Two significant clustering modules (modules A and B) that were identified in the PPI networks. The red nodes represent the upregulated genes and the green nodes represent the downregulated genes. SST, somatostatin precursor; CCL5, chemokine C-C motif ligand 5; CXCL6, chemokine C-X-C motif ligand 6; P2RY14, P2Y purinoceptor 14; ADRA2A, adrenoceptor $\alpha 2 \mathrm{~A}$; C3, complement component 3; GPR18, G-protein-coupled receptor 18; VIM, vimentin; TNNI1, troponin I1; TMOD1, tropomodulin 1; TNNC1, troponin C1; TNNT2, troponin T2; TPM2, tropomyosin $2 \beta$.

Increasing evidence has revealed that in diabetes, there are cytoskeletal changes in podocytes. For instance, advanced glycosylation end products and high glucose can decrease the expression of ACTN4 (40), and a reduced expression of ACTN4 may lead to proteinuria (a symptom of DKD) (41). In addition, FYN is a tyrosine-specific phospho-transferase that belongs to the Src family of tyrosine protein kinases (42). FYN phosphorylation is transiently stimulated by high glucose levels (43). Src/FYN kinase inhibitors disrupt signaling molecules in the VEGF signal transduction pathway (44), and as mentioned above, VEGF may be associated with DKD; thus, FYN may in turn be involved in DKD. In addition, the accumulation of extracellular matrix proteins such as COL1A2 is a key feature of DKD (45). A previous report demonstrated that some key microRNAs (miR) act as effectors of transforming growth factor (TGF) $-\beta$ and the actions of high glucose in DKD (46). In mesangial cells and the kidney, experimental diabetes was associated with the increased expression of COL1A2, and miR-192 was increased by TGF- $\beta$ treatment (45). IGF1 as a growth factor receptor has been associated with type 1 DKD (47). In addition, IGF-1 has the capacity to mediate the histological changes characteristic of DKD (48). These previous studies all indicate that these proteins are associated with DKD. Therefore, the results of the present study are in agreement with these findings and provide further evidence that VEGFA, ACTN4, FYN, COL1A2 and IGF1 may serve important roles in DKD development directly or indirectly. 
In conclusion, the results of the present study indicated that in addition to VEGFA, ACTN4, FYN, COLIA2, IGF1 and HLA-DPAl, immune mechanisms may also serve an important role in the development of DKD. These genes may serve as target genes for the treatment of DKD in future clinical practice. However, this conclusion has no experimental verification; therefore, further evaluation of the potential applications in clinical practice is required.

\section{Acknowledgements}

The present study was supported by the National Natural Science Foundation of China (grant nos. 81070578 and 81270809).

\section{References}

1. Association AD: Standards of medical care in diabetes-2010. Diabetes care 33 (Suppl): S11-S61, 2010.

2. Levin A and Rocco M: KDOQI clinical practice guidelines and clinical practice recommendations for diabetes and chronic kidney disease. Am J Kidney Dis 49 (2 Suppl 2): S12-S154, 2007.

3. Woroniecka KI, Park AS, Mohtat D, Thomas DB, Pullman JM and Susztak K: Transcriptome analysis of human diabetic kidney disease. Diabetes 60: 2354-2369, 2011.

4. Susztak K, Raff AC, Schiffer M and Böttinger EP: Glucose-induced reactive oxygen species cause apoptosis of podocytes and podocyte depletion at the onset of diabetic nephropathy. Diabetes 55 : 225-233, 2006

5. Langham RG, Kelly DJ, Cox AJ, Thomson NM, Holthöfer H, Zaoui P, Pinel N, Cordonnier DJ and Gilbert RE: Proteinuria and the expression of the podocyte slit diaphragm protein, nephrin, in diabetic nephropathy: Effects of angiotensin converting enzyme inhibition. Diabetologia 45: 1572-1576, 2002.

6. Doublier S, Salvidio G, Lupia E, Ruotsalainen V, Verzola D, Deferrari G and Camussi G: Nephrin expression is reduced in human diabetic nephropathy: Evidence for a distinct role for glycated albumin and angiotensin II. Diabetes 52: 1023-1030, 2003.

7. Koop K, Eikmans M, Baelde HJ, Kawachi H, de Heer E, Paul LC and Bruijn JA: Expression of podocyte-associated molecules in acquired human kidney diseases. J Am Soc Nephrol 14: 2063-2071, 2003.

8. Turk T, Leeuwis JW, Gray J, Torti SV, Lyons KM, Nguyen TQ and Goldschmeding R: BMP signaling and podocyte markers are decreased in human diabetic nephropathy in association with CTGF overexpression. J Histochem Cytochem 57: 623-631, 2009.

9. Lindenmeyer MT, Kretzler M, Boucherot A, Berra S, Yasuda Y, Henger A, Eichinger F, Gaiser S, Schmid H, Rastaldi MP, et al: Interstitial vascular rarefaction and reduced VEGF-A expression in human diabetic nephropathy. J Am Soc Nephrol 18 1765-1776, 2007.

10. Hohenstein B, Hausknecht B, Boehmer K, Riess R, Brekken RA and Hugo CP: Local VEGF activity but not VEGF expression is tightly regulated during diabetic nephropathy in man. Kidney Int 69: 1654-1661, 2006.

11. Nath KA: The tubulointerstitium in progressive renal disease. Kidney Int 54: 992-994, 1998.

12. Gilbert RE and Cooper ME: The tubulointerstitium in progressive diabetic kidney disease: More than an aftermath of glomerular injury? Kidney Int 56: 1627-1637, 1999.

13. Strippoli GF, Craig M, Deeks JJ, Schena FP and Craig JC: Effects of angiotensin converting enzyme inhibitors and angiotensin II receptor antagonists on mortality and renal outcomes in diabetic nephropathy: Systematic review. BMJ 329: 828, 2004.

14. Hansen TK, Forsblom C, Saraheimo M, Thorn L, Wadén J, Høyem P, Østergaard J, Flyvbjerg A and Groop PH; FinnDiane Study Group: Association between mannose-binding lectin, high-sensitivity C-reactive protein and the progression of diabetic nephropathy in type 1 diabetes. Diabetologia 53: $1517-1524,2010$

15. Gautier L, Cope L, Bolstad BM and Irizarry RA: affy-analysis of Affymetrix GeneChip data at the probe level. Bioinformatics 20: 307-315, 2004.
16. Ritchie ME, Phipson B, Wu D, Hu Y, Law CW, Shi W and Smyth GK: Limma powers differential expression analyses for RNA-sequencing and microarray studies. Nucleic Acids Res 43: e47, 2015

17. Ashburner M, Ball CA, Blake JA, Botstein D, Butler H, Cherry JM, Davis AP, Dolinski K, Dwight SS, Eppig JT, et al: Gene ontology: Tool for the unification of biology. The Gene Ontology Consortium. Nat Genet 25: 25-29, 2000.

18. Altermann E and Klaenhammer TR: PathwayVoyager: Pathway mapping using the Kyoto Encyclopedia of Genes and Genomes (KEGG) database. BMC Genomics 6: 60, 2005.

19. Wang J, Zhou X, Zhu J, Gu Y, Zhao W, Zou J and Guo Z: GO-function: Deriving biologically relevant functions from statistically significant functions. Brief Bioinform 13: 216-227, 2012.

20. Szklarczyk D, Franceschini A, Kuhn M, Simonovic M, Roth A, Minguez P, Doerks T, Stark M, Muller J, Bork P, et al: The STRING database in 2011: Functional interaction networks of proteins, globally integrated and scored. Nucleic Acids Res 39: D561-D568, 2011.

21. Kohl M, Wiese S and Warscheid B: Cytoscape: Software for visualization and analysis of biological networks. Methods Mol Biol 696: 291-303, 2011.

22. Ichinose K, Kawasaki E and Eguchi K: Recent advancement of understanding pathogenesis of type 1 diabetes and potential relevance to diabetic nephropathy. Am J Nephrol 27: 554-564, 2007.

23. Galkina E and Ley K: Leukocyte recruitment and vascular injury in diabetic nephropathy. J Am Soc Nephrol 17: 368-377, 2006.

24. Chow F, Ozols E, Nikolic-Paterson DJ, Atkins RC and Tesch GH: Macrophages in mouse type 2 diabetic nephropathy: Correlation with diabetic state and progressive renal injury. Kidney Int 65: 116-128, 2004

25. Tuttle KR: Linking metabolism and immunology: Diabetic nephropathy is an inflammatory disease. J Am Soc Nephrol 16: $1537-1538,2005$.

26. Mora $C$ and Navarro JF: Inflammation and diabetic nephropathy. Curr Diab Rep 6: 463-468, 2006.

27. Johnston SL, Virgo PF and Unsworth DJ: Type 1 diabetes mellitus masking primary antibody deficiency. J Clin Pathol 53: 236-237, 2000.

28. Prevoo ML, Van't Hof MA, Kuper HH, Van Leeuwen MA, Van de Putte LB and Van Riel PL: Modified disease activity scores that include twenty-eight-joint counts, development and validation in a prospective longitudinal study of patients with rheumatoid arthritis. Arthritis Rheum 38: 44-48, 1995.

29. Feng PH: Systemic lupus erythematosus: the face of Asia. Ann N Y Acad Sci 1108: 114-120, 2007.

30. Varney MD, Valdes AM, Carlson JA, Noble JA, Tait BD, Bonella P, Lavant E, Fear AL, Louey A, Moonsamy P, et al: HLA DPA1, DPB1 alleles and haplotypes contribute to the risk associated with type 1 diabetes: Analysis of the type 1 diabetes genetics consortium families. Diabetes 59: 2055-2062, 2010.

31. Santin I, Castellanos-Rubio A, Aransay AM, Gutierrez G, Gaztambide S, Rica I, Vicario JL, Noble JA, Castaño L and Bilbao JR: Exploring the diabetogenicity of the HLA-B18-DR3 $\mathrm{CEH}$ : Independent association with T1D genetic risk close to HLA-DOA. Genes Immun 10: 596-600, 2009.

32. Dvorak HF, Brown LF, Detmar M and Dvorak AM: Vascular permeability factor/vascular endothelial growth factor, microvascular hyperpermeability, and angiogenesis. Am J Pathol 146: 1029-1039, 1995

33. Schrijvers BF, Flyvbjerg A and De Vriese AS: The role of vascular endothelial growth factor (VEGF) in renal pathophysiology. Kidney Int 65: 2003-2017, 2004.

34. Baelde HJ, Eikmans M, Doran PP, Lappin DW, de Heer E and Bruijn JA: Gene expression profiling in glomeruli from human kidneys with diabetic nephropathy. Am J Kidney Dis 43: 636-650, 2004.

35. Bortoloso E, Del Prete D, Dalla Vestra M, Gambaro G, Saller A, Antonucci F, Baggio B, Anglani F and Fioretto P: Quantitave and qualitative changes in vascular endothelial growth factor gene expression in glomeruli of patients with type 2 diabetes. Eur J Endocrinol 150: 799-807, 2004.

36. Cha DR, Kim NH, Yoon JW, Jo SK, Cho WY, Kim HK and Won NH: Role of vascular endothelial growth factor in diabetic nephropathy. Kidney Int Suppl 77: S104-S112, 2000.

37. Eremina V, Sood M, Haigh J, Nagy A, Lajoie G, Ferrara N, Gerber HP, Kikkawa Y, Miner JH and Quaggin SE: Glomerular-specific alterations of VEGF-A expression lead to distinct congenital and acquired renal diseases. J Clin Invest 111: $707-716,2003$. 
38. Caldwell RB, Bartoli M, Behzadian MA, El-Remessy AE, Al-Shabrawey M, Platt DH and Caldwell RW: Vascular endothelial growth factor and diabetic retinopathy: Pathophysiological mechanisms and treatment perspectives. Diabetes Metab Res Rev 19: 442-455, 2003.

39. Nikolopoulos SN, Spengler BA, Kisselbach K, Evans AE, Biedler JL and Ross RA: The human non-muscle alpha-actinin protein encoded by the ACTN4 gene suppresses tumorigenicity of human neuroblastoma cells. Oncogene 19: 380-386, 2000.

40. HA TS: High glucose and advanced glycosylated end-products affect the expression of alpha-actinin-4 in glomerular epithelial cells. Nephrology (Carlton) 11: 435-441, 2006.

41. Jefferson JA, Shankland SJ and Pichler RH: Proteinuria in diabetic kidney disease: A mechanistic viewpoint. Kidney Int 74: 22-36, 2008.

42. Resh MD: Fyn, a Src family tyrosine kinase. Int J Biochem Cell Biol 30: 1159-1162, 1998.

43. Taniguchi K, Xia L, Goldberg HJ, Lee KW, Shah A Stavar L, Masson EA, Momen A, Shikatani EA, John R, et al: Inhibition of Src kinase blocks high glucose-induced EGFR transactivation and collagen synthesis in mesangial cells and prevents diabetic nephropathy in Mice. Diabetes 62: 3874-3886, 2013.
44. van Nieuw Amerongen GP and van Hinsbergh VW: Targets for pharmacological intervention of endothelial hyperpermeability and barrier function. Vascular pharmacol 39: 257-272, 2002.

45. Kato M, Zhang J, Wang M, Lanting L, Yuan H, Rossi JJ and Natarajan R: MicroRNA-192 in diabetic kidney glomeruli and its function in TGF-beta-induced collagen expression via inhibition of E-box repressors. Proc Natl Acad Sci USA 104: 3432-3437, 2007.

46. Kato M, Arce L and Natarajan R: MicroRNAs and their role in progressive kidney diseases. Clin J Am Soc Nephrol 4: 1255-1266, 2009.

47. Ewens KG, George RA, Sharma K, Ziyadeh FN and Spielman RS: Assessment of 115 candidate genes for diabetic nephropathy by transmission/disequilibrium test. Diabetes 54: 3305-3318, 2005.

48. Schreiber B, Hughes M and Groggel G: Insulin-like growth factor-1 stimulates production of mesangial cell matrix components. Clin Nephrol 43: 368-374, 1995. 